\title{
Relación entre el nivel de conocimiento con el grado de cumplimiento de las precauciones estándar de bioseguridad del personal técnico de enfermería en Ios servicios de Neurología y Neurocirugía del Instituto Nacional de Ciencias Neurológicas
}

\author{
Relationship between the level of knowledge with the degree of compliance with standard precautions \\ biosafety staff nursing services for Neurology and Neurosurgery, National Neuroscience Institute.
}

Felicitas Quispe Masías', Sara Beatriz Huanca Flores², Gladys Carmela Ramos Medina

\begin{abstract}
RESUMEN
Objetivo: Determinar la relación existente entre el nivel de conocimiento y el grado de cumplimiento de las precauciones estándar de bioseguridad del personal técnico de enfermería de los servicios de Neurología y Neurocirugía del Instituto Nacional de Ciencias Neurológicas, octubre 2011 a marzo del 2012. Material y Métodos: El estudio fue de tipo cuantitativo, correlacional y transversal. La muestra estuvo conformada por 51 técnicos de enfermería del Instituto Nacional de Ciencias Neurológicas, seleccionada mediante muestreo no probabilístico, por conveniencia. La información fue generada a través de un cuestionario y una guía de observación elaborados por los autores. Ambos instrumentos fueron sometidos al juicio de expertos y a la prueba de confiabilidad según el coeficiente de Kudder Richardson (0,77). Resultados: Los resultados muestran que el $64.7 \%$ de los técnicos de enfermería poseen un nivel de conocimientos medio sobre las precauciones estándar de bioseguridad, el $23.5 \%$ posee un nivel bajo y solo el $12 \%$ un nivel alto. En relación al nivel de cumplimiento de las precauciones estándar de bioseguridad, se encuentra que el $43.1 \%$ tiene un nivel medio, el $35.2 \%$ tiene un nivel alto y un $21.5 \%$ un nivel bajo. Conclusiones: Según la prueba no paramétrica de Pearson, se encontró correlación entre ambas variables $(0,068)$ siendo significativa $(p>$ de 0,05$)$.
\end{abstract}

Palabras clave: Conocimiento, cumplimiento, bioseguridad y técnicos de enfermería.

\section{SUMMARY}

Objective: To determine the relationship between the level of knowledge and degree of compliance with standard precautions biosafety staff nursing services for Neurology and Neurosurgery, National Neuroscience Institute, October 2011 to March 2012. Material and Methods: The study was quantitative, correlational and crosssectional. The sample was composed of 51 nursing technicians of the National Institute of Neurological Sciences, selected by non-probability sampling, for convenience. The information was generated through a questionnaire and an observation guide elavorados by the authors. Both instruments were subjected to the judgment of experts and the reliability test under Kudder Richardson coefficient ( 0.77). Results: The results show that $64.7 \%$ of nursing technicians have an average level of knowledge standard biosafety precautions, $23.5 \%$ have a low level and only $12 \%$ a high level. Regarding the level of compliance with standard precautions biosecurity, which is $43.1 \%$ a medium level, $35.2 \%$ have a high level and $21.5 \%$ low. Conclusions: According to the nonparametric Pearson correlation was found between the two variables (0.068) being significant ( $p>0.05)$.

Keywords: Knowledge, compliance, biosafety and nursing technicians.

\footnotetext{
${ }^{1}$ Enfermera asistencial en el servicio de cirugía del Instituto Nacional de Ciencias Neurológicas, Lima, Perú.

${ }^{2}$ Enfermera asistencial en la Clínica Good Hope, Miraflores, Perú.

${ }^{3}$ Enfermera asistencial en el servicio de cirugía del Instituto Nacional de Ciencias Neurológicas, Lima, Perú.
} 


\section{INTRODUCCIÓN}

La seguridad es un componente básico de la calidad. De su adecuada observancia depende tanto la oportuna recuperación del paciente al no verse complicado por elementos adicionales, como el bienestar del propio personal (Malagón Londoño, Galán y Pontón 2000).

La estancia prolongada por complicaciones intrahospitalarias, los periodos de incapacidad debido a accidentes punzocortantes e infecciones contraídas por no usar las medidas de protección y el efecto deletéreo de las demandas legales, es solo la punta del iceberg de los efectos relacionados con el desconocimiento y la inobservancia de las medidas de bioseguridad en el medio asistencial (Harada 2006).

Desde el año 2006 en el Perú, el personal directivo del Ministerio de Salud, toma conciencia de la importancia de lograr la implementación de normas y protocolos para asegurar las mejores prácticas de bioseguridad y la protección deseada para el personal.

EsSalud reportó, entre el 2005-2007, un importante incremento de contagio de Tuberculosis Pulmonar de tipo ocupacional, identificando que curiosamente en los servicios donde el riesgo era evidente, no existía casos de contagio ocupacional, mientras en servicios generales, Emergencia, Laboratorio y aún en la UCI, los casos eran frecuentes. Oficina de Inteligencia Sanitaria de Lima.

El conocimiento y adherencia a las buenas prácticas de bioseguridad, es una parte importante del control del riesgo, al que todo el personal asistencial está expuesto. El personal técnico de enfermería, en particular, no solo se expone en el contacto con el paciente, sino en la toma y trasporte de muestras de diferentes tipos, en los procesos de descontaminación de material utilizado en procedimientos invasivos (MINSA 2010).

En base a las premisas expuestas cabe señalar que, durante las dos últimas décadas, se ha enfatizado la corresponsabilidad existente entre quienes gerencian las instituciones sanitarias y el propio trabajador, quien tiene las herramientas y la capacitación necesaria para autoprotegerse.

Sin embargo, en la práctica cotidiana, es un hecho generalizado, que existe baja adherencia a las medidas básicas, llamadas precauciones estándar de bioseguridad, las mismas que constituyen un conjunto de medidas aplicables a diferentes espacios de riesgo (EsSalud 2003).

Es frecuente observar al personal con la mascarilla ya usada, colgando sobre el cuello; sentarse a escribir en los registros del servicio, sin quitarse el guardapolvo o mandil protector usado durante la asistencia directa del paciente; omitir la higiene de manos antes y después de atender al paciente, comportamientos que conducen a interrogantes: ¿Qué conocimientos tiene el personal acerca de las precauciones estándar con sangre y fluidos corporales? ¿Qué grado de acatamiento tiene ante las Buenas Prácticas en este campo?

La bioseguridad es el conjunto de medidas preventivas que tiene como objetivo principal proteger la salud, seguridad del personal y pacientes frente a diversos riesgos ocasionados por agentes biológicos, químicos, físicos y mecánicos; estas normas nos indican cómo hacer para cometer menos errores y sufrir menos accidentes (MINSA 2010).

El conocimiento y la práctica de las precauciones universales comprende un conjunto de medidas de barrera que son suficientes para prevenir la exposición cutánea o mucosa, cuando entra en contacto con sangre y/o fluidos corporales de pacientes; abarca el lavado de manos, mascarillas, guantes, mandilón clínico, gorro, botas, protector ocular, desechos de material punzocortante en cajas especiales y manejo de ropa sucia.

El personal técnico debe tener suficiente información sobre las medidas y precauciones estándar de bioseguridad, ya que la inadecuada práctica de las normas, pueden conducir a un incremento de los costos, prolongación de la estancia hospitalaria y complicaciones en el paciente. El objetivo del estudio es determinar el nivel de conocimientos con el grado de cumplimiento de las precauciones estándar de bioseguridad del personal técnico de enfermería en los servicios de neurología y neurocirugía del Instituto Nacional de Ciencias Neurológicas.

\section{MATERIAL Y MÉTODOS}

La investigación fue de tipo observacional, porque se estudió el comportamiento de las variables tal como se produce en la realidad. Según el alcance de los resultados, fue descriptivo, correlacional y según las mediciones realizadas en un momento dado, fue de corte transversal.

La sede fue el Instituto Nacional de Ciencias Neurológicas, con la participación de técnicos de enfermería que laboran en áreas de hospitalización, durante los meses de octubre 2011 a marzo 2012. La muestra estuvo constituida por 51 técnicos equivalente al total de la población, por lo que el muestreo fue no probabilístico por conveniencia para el estudio.

Para la obtención de los datos, se diseñó un cuestionario y una lista de cotejo de las prácticas a observar en el personal técnico; el cuestionario fue 
Relación entre el nivel de conocimiento y cumplimiento de las precauciones estándar de bioseguridad del personal técnico de enfermería en los servicios de Neurología y Neurocirugía del Instituto Nacional de Ciencias Neurológicas

validado según el Coeficiente biserial puntual siendo de 0,70 y la lista de cotejo según el coeficiente KR de Kudder Richardson tuvo una confiabilidad de 0.77 , es decir aceptable confiabilidad.

Luego de obtener la autorización oficial para ejecutar el proyecto, se coordinó con la jefatura del servicio. Del mismo modo, se solicitó y obtuvo la aceptación de los sujetos informantes, los técnicos de enfermería.
Se aplicó los instrumentos según cronograma, entre el 10 y el 20 de febrero 2012.

Finalmente se procesó la información distribuyendo tanto el nivel de conocimientos como el grado de cumplimiento de las precauciones estándar, mediante la escala de Stanimos, para su posterior análisis e interpretación.

\section{RESULTADOS}

\section{Tabla 1}

Técnicos de enfermería según características generales en el servicio de neurocirugía del Instituto Nacional de Ciencias Neurológicas. Lima, 2012.

\begin{tabular}{lcc}
\hline Características & $\mathrm{n}$ & $\%$ \\
\hline Sexo & & \\
Masculino & 10 & 19.6 \\
Femenino & 41 & 81.39 \\
Tiempo de servicio & & \\
1 a 3 años & 7 & 13.72 \\
4 a 6 años & 7 & 13.72 \\
7 años a más & 37 & 72.56 \\
Capacitación en bioseguridad & & \\
Sí recibió & 49 & 90 \\
No recibió & 2 & 10 \\
\hline
\end{tabular}

La muestra de estudio fue un grupo de técnicos a predominio femenino $(81.39 \%)$, con un tiempo de servicios de 7 a más años y con capacitación en bioseguridad en un $90 \%$.

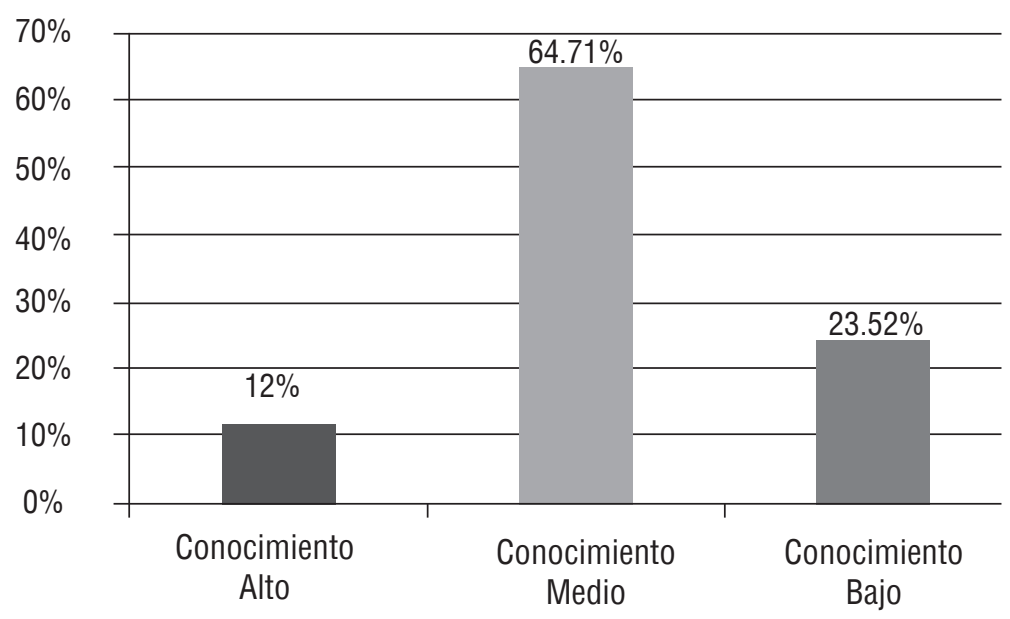

Figura 1

Técnicos de enfermería según nivel de conocimientos sobre precauciones estándar de bioseguridad en los servicios de neurología y neurocirugía del Instituto Nacional de Ciencias Neurológicas, 2012. 
Del total de técnicos de enfermería $(51=100 \%)$, se encontró el porcentaje más alto en el nivel de conocimiento medio $(64,7 \%)$ seguido del nivel de conocimiento bajo $(23,5 \%)$.

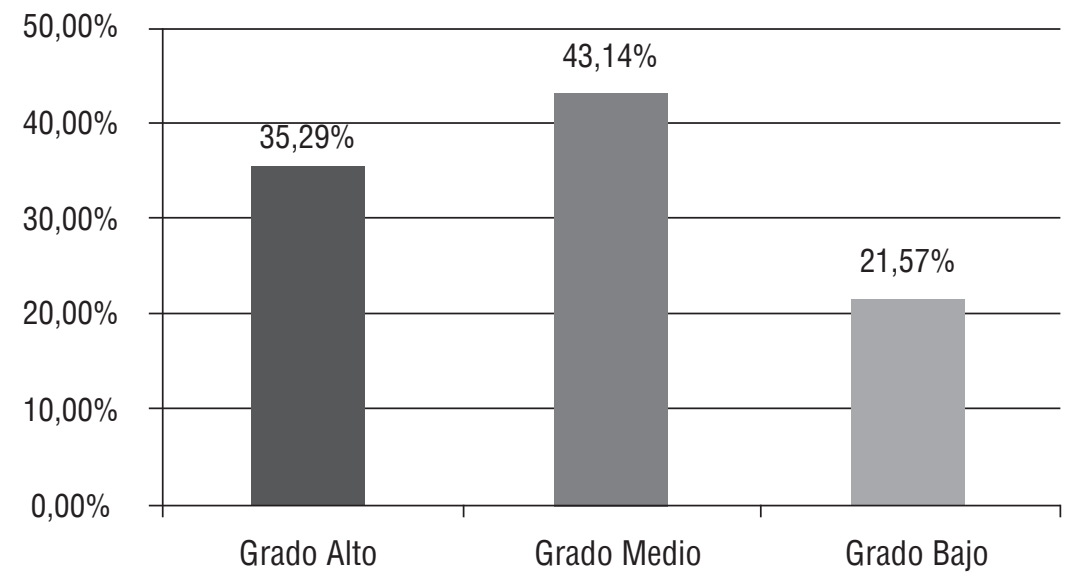

Figura 2

Técnicos de enfermería según grado de cumplimiento de las precauciones estándar de bioseguridad en los servicios de neurología y neurocirugía del Instituto Nacional de Ciencias Neurológicas de octubre 2011- marzo 2012.

Del total de técnicos de enfermería, se encontró el mayor porcentaje en el grado medio de cumplimiento de las precauciones estándar de bioseguridad (43.1\%), seguido del grado alto de cumplimiento en un $35.2 \%$.

Tabla 2

Análisis de correlación entre conocimientos y el grado de cumplimiento de las precauciones estándar de bioseguridad en técnicos de enfermería.

\begin{tabular}{lll}
\hline & $r$ & $p$ \\
\hline $\begin{array}{l}\text { Conocimientos en } \\
\text { precauciones Estándar }\end{array}$ & .068 & .058 \\
$\begin{array}{l}\text { Grado de Cumplimiento } \\
\text { de precauciones }\end{array}$ & .068 & .258 \\
Estándar & & \\
\hline
\end{tabular}

Del análisis estadístico, según la prueba no paramétrica de Pearson, se concluye que existe baja correlación entre ambas variables (0.068) siendo significativa $(\mathrm{p}>0.05)$.

\section{DISCUSIÓN}

Los hallazgos del estudio reportan conocimientos en nivel medio sobre las precauciones estándar de bioseguridad. En su contenido, guardan similitud con los estudios realizados por Alvarado (2004), Fiestas, Gambini, y Montesinos (2005), quienes encontraron nivel medio de conocimientos y aplicabilidad de las normas de bioseguridad, tanto en enfermeras como en técnicos de enfermería, evidenciando menor adherencia en el uso de lentes protectores, las uñas cortas y el desecho de materiales punzocortantes en cajas adecuadas.

Los resultados obtenidos respecto al grado de cumplimiento de las precauciones estándar de bioseguridad por parte de los técnicos de enfermería, también se ubican en el nivel medio. Estos resultados requieren atención oportuna, pues existe el riesgo de bajar a un nivel menor y traer consecuencias adversas en el cuidado de los pacientes neurológicos. Por tanto, se debe fortalecer estas prácticas mediante estímulo y educación continua coincidiendo con Roschke y Casas (1987 citados por Davini, 1995), cuando señala que sin educación continua y una 
Relación entre el nivel de conocimiento y cumplimiento de las precauciones estándar de bioseguridad del personal técnico de enfermería en los servicios de Neurología y Neurocirugía del Instituto Nacional de Ciencias Neurológicas

fuente de motivación, las competencias laborales decrecen inexorablemente como consecuencia de innumerables factores individuales y organizativos que comprenden el incremento de la demanda de cuidado y las limitaciones en la tecnología requerida.

Aburto (2012), manifiesta que detrás de la falta de correspondencia entre el conocimiento y la práctica de bioseguridad, se encuentra los problemas de actitud, una forma de resistencia activa y pasiva a respetar las normas existentes en bioseguridad, motivadas por un exceso de confianza en las fuerzas propias o falta de credibilidad en prácticas sostenidas en el tiempo

Restrepo y Málaga (2000), señalan que para lograr congruencia entre el conocimiento y la práctica de las precauciones estándar de bioseguridad, es necesario llevar al trabajador al plano reflexivo, donde sea capaz de formar una conciencia crítica de la realidad acerca del alto riesgo biológico, y crear compromiso para la acción transformadora y sostenida.

Malangón, Galán y Pontón (2001), expresan la importancia que las instituciones deben conceder no solo a la enseñanza, sino al monitoreo de los resultados de la misma. Agregan a tal fin que el verdadero esfuerzo debe situarse en lograr que se integre, en el proyecto de educación permanente, la revisión de una cultura de trabajo y los valores subyacentes en la práctica asistencial; esta modalidad conlleva a un enriquecimiento personal que compromete al personal en el cambio.

La baja correlación identificada entre las variables de interés explica el importante papel que juega el aspecto cultural; lograr cambios favorables en el comportamiento del personal, requiere la progresiva y perseverante incorporación de los conocimientos sobre el riesgo y las prácticas para minimizarlo, en el conjunto de valores de los miembros de un establecimiento de salud, de manera que pase a formar parte de la cultura organizacional hasta lograr la llamada cultura de seguridad (Harada, 2006).

No obstante, ser una necesidad largamente sentida por directivos y personal operativo y de tanto valor para la vida humana que es materia de legislación sobre seguridad y salud en el trabajo, los resultados expresan la larga trayectoria por recorrer hasta lograr un compromiso significativo en las esferas cognitiva, afectiva y volitiva del personal de enfermería y aun, de todo el equipo de salud.

Dados los resultados obtenidos, con evidente baja correlación entre el conocimiento y la práctica de precauciones estándar de bioseguridad, es posible concluir que es necesario desplegar mayores esfuerzos de capacitación hasta lograr mantener adecuados niveles de apropiación del saber tecnológico destinado a prevenir y controlar la diseminación de infecciones en un contexto laboral de alto riesgo.

\section{Declaración de financiamiento y de conflicto de intereses:}

El estudio fue financiado por la autora, quien declara no tener algún tipo de conflicto de interés en la investigación realizada.

\section{Correspondencia:}

Felicitas Quispe Masías

Instituto Nacional de Ciencias Neurológicas.

Correo electrónico: margaritafeli@hotmail.com 


\section{REFERENCIAS BIBLIOGRÁFICAS}

Aburto y (2012). Gestión de los riesgos asistenciales, conferencia congreso de tratamiento avanzado de heridas. Sheraton, Lima-Perú.

Alvarado Reyes N. (2005). Nivel de conocimiento y grado de aplicabilidad de las Normas de bioseguridad por el equipo de enfermería del Hospital de Sullana.

Cuyubamba (2003). "Conocimiento y Aplicación de las Medidas de Bioseguridad por el Personal de Salud". Tesis de Bachiller en el Hospital "Félix Mayorca Soto". Tarma.

EsSalud (2003). Contaminación ocupacional por Tuberculosis. Factores relacionados. Gerencia Central de Recursos Humanos. Lima.

EsSalud (2007). Adherencia a las Precauciones Universales con Sangre y fluidos corporales, Oficina de Inteligencia Sanitaria. Lima.

Davini C. (1995). Educación permanente en salud. En: Organización Panamericana de la Salud. Publicación cientifica $\mathrm{N} .{ }^{\circ} 38$ Washington D.C.
Harada M. (2006). Seguridad del paciente. Ed. Innovación. Brasil.

Le Mone P. y Burke, K. (2009). Enfermería médico quirúrgico. (4a ed.Vol.1) Editorial Pearson Prentice Hall. España.

Malagón, L., Galán M. y Pontón L. (2000). Administración hospitalaria (2da ed.). Editorial Panamericana, Colombia.

Red Asistencial Almenara (2010) Reglamento interno de salud en el trabajo, EsSalud-Lima.

MINSA (2010). Plan Nacional de Seguridad del Paciente. Lima-Perú.

Restrepo H. y Málaga H. (2001). Promoción de la salud. Editorial Médica. Panamericana. Colombia.

Soto B. et al (2010). Conocimiento y cumplimiento de medidas de bioseguridad en el personal de enfermería del Hospital Nacional Almanzor Aguinaga- Chiclayo.

Recibido: 7/04/2013 Aceptado: 16/09/2013 